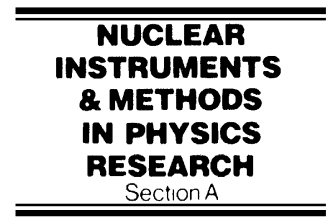

www.elsevier.com/locate/nima

\title{
Luminescence and imaging with gas electron multipliers
}

\author{
F.A.F. Fraga ${ }^{\mathrm{a}, \mathrm{b}, *}$, L.M.S. Margato ${ }^{\mathrm{a}}$, S.T.G. Fetal ${ }^{\mathrm{a}}$, M.M.F.R. Fraga ${ }^{\mathrm{a}, \mathrm{b}}$, \\ R. Ferreira Marques ${ }^{\mathrm{a}, \mathrm{b}}$, A.J.P.L. Policarpo ${ }^{\mathrm{a}, \mathrm{b}}$ \\ ${ }^{a}$ LIP_Coimbra, Departamento de Física, Universidade de Coimbra, Coimbra 3004-516, Portugal \\ ${ }^{\mathrm{b}}$ CFRMUC-Centro de Física, Radiação e de Materiais, Departamento de Física da, Universidade de Coimbra, \\ Coimbra 3004-516, Portugal
}

\begin{abstract}
Although the GEM has been mainly used as a gaseous preamplifier device, it has been shown that using suitable gaseous mixtures the avalanches emit a large number of photons, in the UV, visible and/or NIR bands. This scintillation, readout by CCDs, has been used recently for the development of imaging detectors to be used with X-rays, alpha particles and neutrons. The luminescence process in the GEM and a summary of its applications will be presented. The latest developments, including the operation of the GEMs in photon counting mode, will also be referred.
\end{abstract}

(C) 2003 Elsevier B.V. All rights reserved.

PACS: 29.40.Cs; 29.40.Gx; 29.40.Mc

Keywords: GEM detectors; CCD; Microstructures; Scintillation

\section{Introduction}

The advantages of optical readout of detectors are well known: the electronics are decoupled from the detection media, becoming insensitive to electronic noise or RF pickup signals coming from the detector media and housing [1], they can cover large areas without dead spaces using optical systems such as lenses, mirrors, fibers and tapers, and, it should be emphasized, they are particularly suited for recording complex events when using

\footnotetext{
*Corresponding author. Address for correspondence: LIPCoimbra, Departmento de Física, Universidade de Coimbra, Coimbra 3000, Portugal. Tel.: + 351-39-833-465; fax: +351-39822-358.

E-mail address: francisco@lipc.fis.uc.pt (F.A.F. Fraga).
}

true pixel readouts, making possible real multihit capability. 2D imaging detectors using gas scintillation with optical electronic readout have been widely used in Physics experiments during the last 30 years, but the idea of using the light pulses produced by the avalanches in proportional counters was suggested by Charpak as early as 1956 [2] and the earliest 2D gas scintillators with optical readout by PMs or intensified CCDs were initially used with wires and pure gases such as Xe, $\mathrm{Kr}$, Ar and $\mathrm{He}$ with the addition of $\mathrm{N}_{2}$ (see Fig. 1) [3]. These gases scintillate below $200 \mathrm{~nm}$ and require the use of inefficient and expensive optics or optical wavelength shifters to couple the electronic optical readout to the detector.

Later improvements, such as continuous amplifying structures (PPAC, grids) and the use of gas 


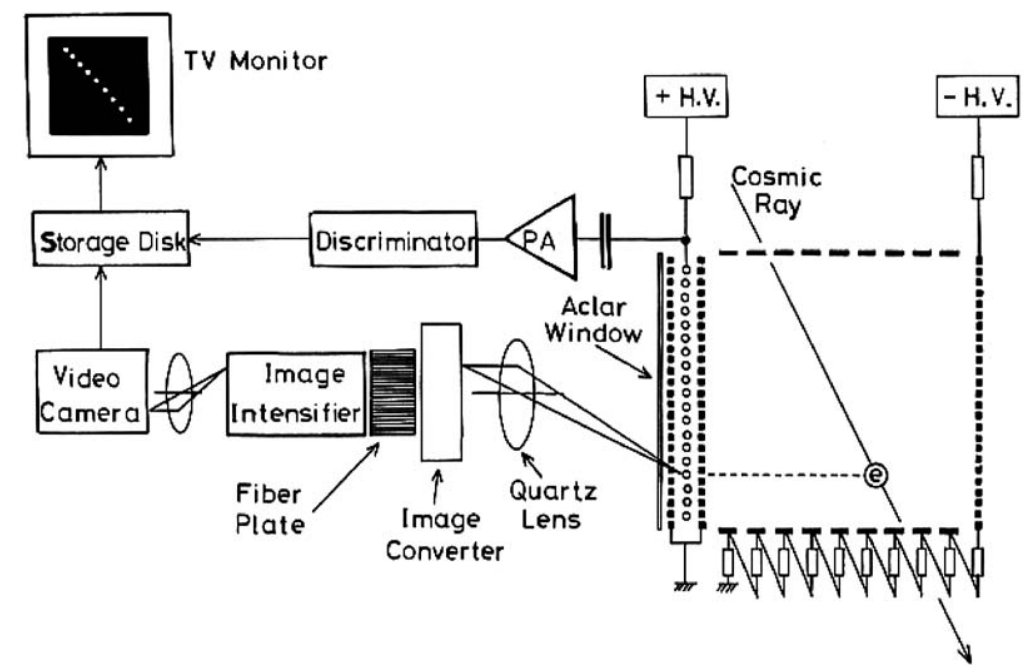

Fig. 1. Schematic of an optical readout gas avalanche chamber presented in 1988. The drift volume is coupled to a MWPC sensing element.

mixtures scintillating at $\lambda>250 \mathrm{~nm}$, extended the application of these detectors [4-6]. But some limiting features remained, mainly the low number of emitted photons, requiring the use of image intensifiers that are expensive, have limited size and degrade image resolution. Although this limitation could be addressed using large scintillation gaps, on account of the electron diffusion and the optical depth of field they degrade position resolution. Some interesting, but technically complicated and expensive solutions using low pressure, high-temperature gas mixtures and special devices as capillary plates were later presented $[7,8]$, but could not completely address the limitations of these detectors.

In 1993, Oed and Geltenbort [9] reported high luminosity from rare gas mixtures of $\mathrm{Ar}, \mathrm{Xe}$ and $\mathrm{Kr}$ using microstrips irradiated with a ${ }^{55} \mathrm{Fe}$ source, quoting 60 times more light than from a microstrip operated at a gain of $4 \times 10^{4}$ from a $1 \mathrm{~mm}$ thick NaI (П) scintillator with identical PM and electronics. Our group used the scintillation of avalanches readout by a CCD with a spectral sensitivity between 400 and $1000 \mathrm{~nm}$ to perform quality control of microstrips in 1998 (Fig. 2), and later, after Sauli presented a new microstructure, the GEM (gas electron multiplier) [10], we started a program on the study and development of

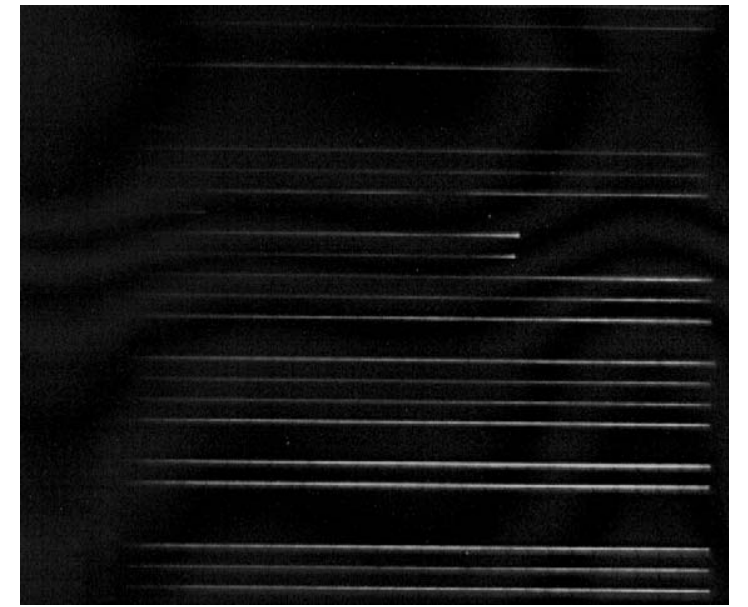

Fig. 2. CCD image of the scintillation observed on a damaged ILL6C microstrip plate under X-ray illumination.

detectors using GEMs as "active scintillators", as first denominated by Oed.

\section{The GEM scintillation}

The gas electron multiplier is a thin insulating foil, metal clad on both sides and perforated by a regular dense matrix of holes. Full details about its manufacturing and mode of operation can be 


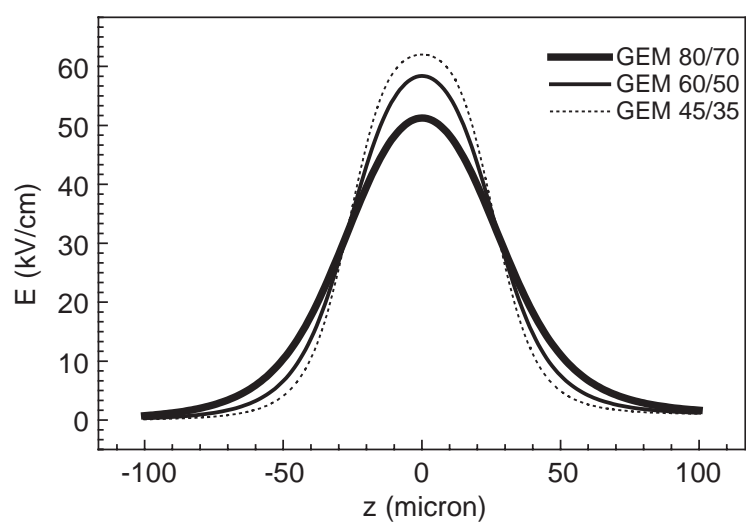

Fig. 3. Magnitude of the electric field along the center of the GEM channel for equal measured gain in GEMs of different metal hole size.

found elsewhere [11], we will only mention that in typical operation the intense electrical field in the multiplication hole has a magnitude of some tens of $\mathrm{kV} / \mathrm{cm}$ along a distance of some tens of microns (Fig. 3) and the electrons entering inside this hole experience a very localized multiplication. If a proper gas mixture is chosen the excited species can scintillate emitting photons along a very short path also. Considering that the GEM is optically transparent almost half of these photons exit the GEM hole through the channel and can be detected by a readout without any loss or shadow due to the multiplication element. Since the GEM has high gain, the number of emitted photons per incoming electron can be very high, and as the emission is localized in the thin GEM gap, there is no blurring of images originated by depth of field effects. Also, the high intensity and configuration of the field suggest that gas mixtures with higher excitation energy, scintillating at the visible and NIR bands of the spectrum, could be used for the development of active scintillators.

The system we have used to study the GEM scintillation is shown in Fig. 4. It is fully described in Ref. [12]. We only mention a few aspects. Standard GEMs with an area of $5 \times 5 \mathrm{~cm}^{2}$ having 80 and $60 \mu \mathrm{m}$ metal diameter holes with, respectively, 70 and $50 \mu \mathrm{m}$ biconical kapton holes were used and an optically transparent collecting grid was placed $2 \mathrm{~mm}$ far from the second GEM, defining the induction gap. A photomultiplier

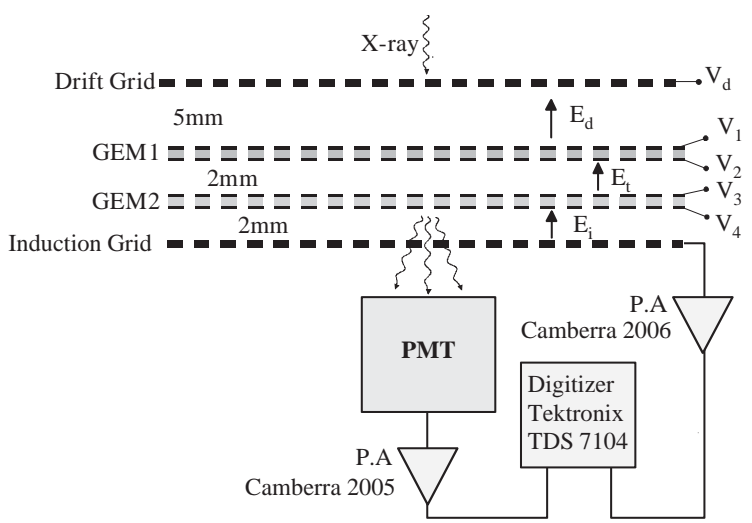

Fig. 4. The avalanche scintillation light was read by a photomultiplier Philips 56TUVP-type B. A Tektronix TDS 7104 scope $(1 \mathrm{GHz}, 10 \mathrm{GS} / \mathrm{s})$ was used to digitize the light and charge signals.

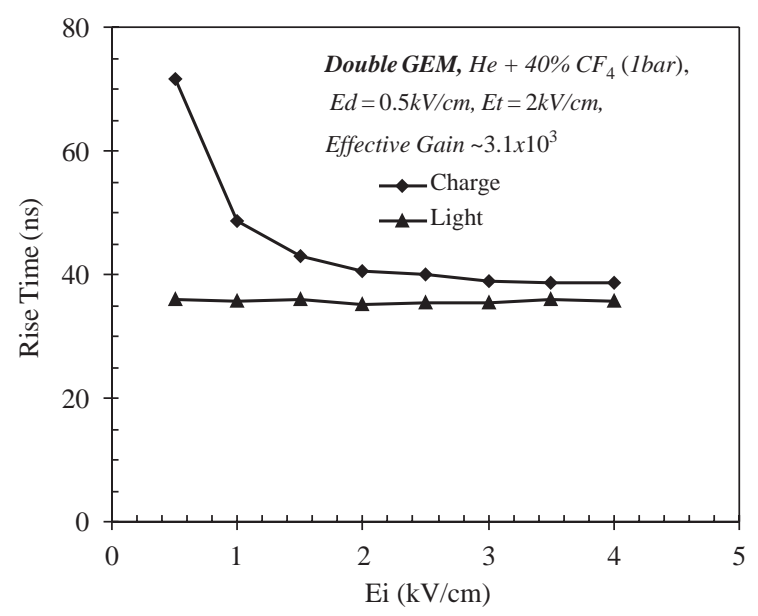

Fig. 5. Average rise time of the light and charge signals of a double GEM system versus induction field, $E_{i}$.

(Philips 56TUVP-type B-extended S20) has been coupled to the quartz window of the detector and the charge and light signals were readout by Camberra 2005 and Camberra 2006 preamplifiers, respectively. A Tektronix TDS 7104 scope (1 GHz, $10 \mathrm{GS} / \mathrm{s}$ ) was used to simultaneously digitize the light and charge signals at the output of the preamplifiers. Fig. 5 shows the variation of the light and charge signals versus induction field, measured using a $\mathrm{He}-\mathrm{CF}_{4}$ mixture and a ${ }^{55} \mathrm{Fe}$ source. As expected, the light signals do not depend on the induction field and are faster than the charge signals. The energy resolution of both 


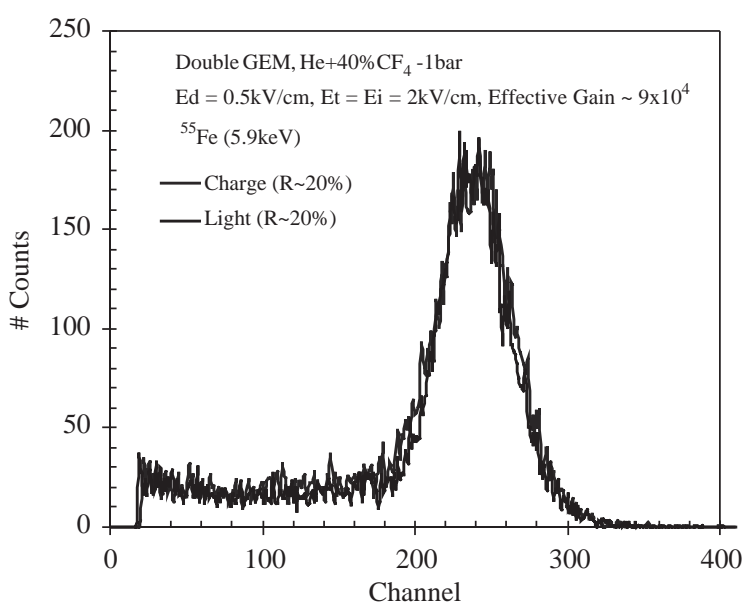

Fig. 6. Energy resolution using the light and charge signals measured with a double GEM system.

type of signals is shown in Fig. 6, showing clearly that light signals can be used for gating purposes in multiple GEM systems, as done before in PPACs.

\section{GEMs and CCDs}

Immediately after the start we considered the possibility of using the GEM scintillation readout by a CCD camera to check for local defects and gain uniformity of the GEM foils. Considering the typical sensitivity of visible light CCDs (q.e. $>10 \%$ between 400 and $1000 \mathrm{~nm}$ ) we found that the atomic emission lines of Ar would be suitable for this purpose and that the addition of $5 \% \mathrm{CO}_{2}$ would be a suitable compromise between mixture stability and luminosity, with a light yield $\sim 0.03$ photons/secondary electron. Fig. 7 shows that the scintillation is very sensitive to electric field configuration and we could even find defects unseen with an optical inspection system from the CCD images [13].

\section{Imaging chambers}

Several other characteristics, other than the high electrical field inside the holes, render the GEM very attractive for imaging scintillators. GEMs can

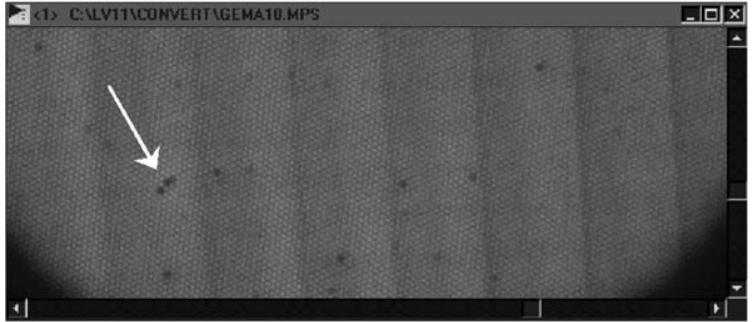

(a)

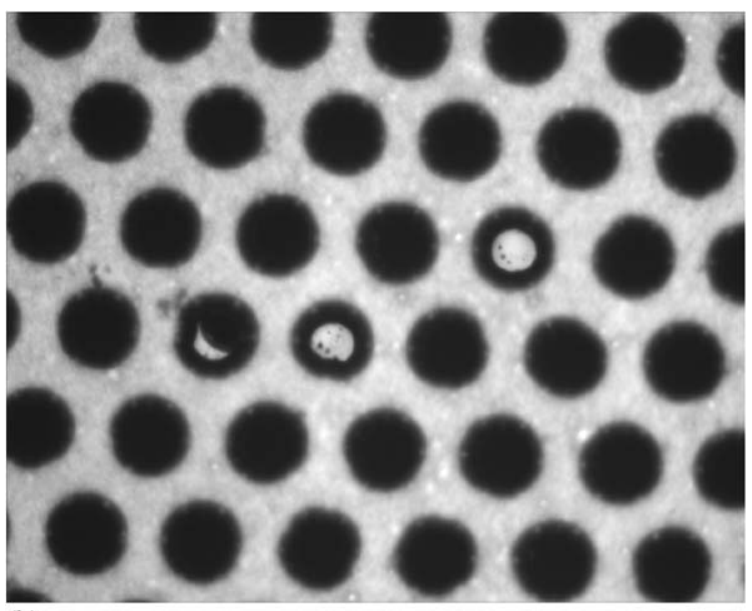

(b)

Fig. 7. Scintillation image of a GEM area and detail of the GEM foil area indicated by the arrow taken.

be cascaded for high gain (up to $10^{6}$ ) using up to 4 stages $[14,15]$. They are free from ion feedback as most of the ions produced in a GEM hole are collected in the previous GEM, avoiding high positive space charges in the interaction volume [16] and, because of the photon screening due to their low hole to surface ratio, are intrinsically free from photon feedback [17]. Also, when readout in light mode, there is no need to collect the electrons on the induction electrode avoiding breakdown in the last stage. GEMs can be manufactured in large areas $\left(\sim 30 \times 30 \mathrm{~cm}^{2}\right)[18]$ and have high ganularity_ pitch can be as low as $90 \mu \mathrm{m}$.

Once a scintillation gas mixture with a much higher scintillation yield $(\sim 0.7 \quad$ photons per secondary electron) having adequate emission spectra was found ( $\mathrm{Ar}-\mathrm{CF}_{4}$ see Fig. 8) [19], the idea of developing an imaging chamber ensued. We describe here a tracking chamber built with GEMs that has been used for imaging typical 


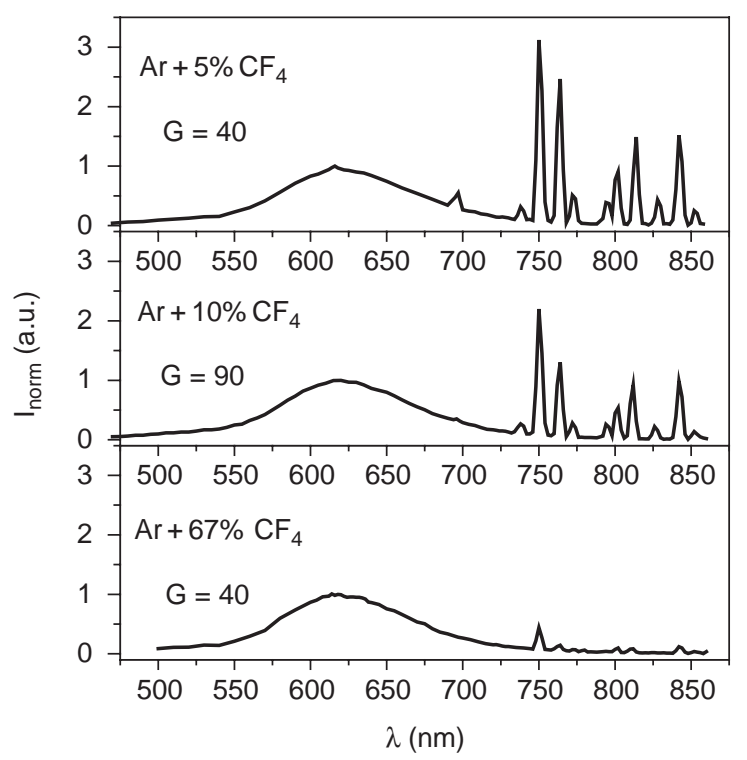

Fig. 8. Visible and NIR emission spectra of $\mathrm{Ar}-\mathrm{CF}_{4}$ mixtures, normalized to the light intensity at $620 \mathrm{~nm}$.

energy deposits of alpha particles in gas media. The chamber has a sensitive volume of $\sim 250 \mathrm{~cm}^{3}$ and can image track lengths up to $8 \mathrm{~cm}$.

The schematic drawing of the chamber is shown in Fig. 9. The chamber was fitted with three $10 \times 10 \mathrm{~cm}^{2}$ cascaded GEMs, separated by $2 \mathrm{~mm}$, and a collecting electrode placed $3 \mathrm{~mm}$ away from the outer GEM and was filled with $\mathrm{Ar}-5 \% \mathrm{CF}_{4}$. The CCD camera was placed at $30 \mathrm{~cm}$ from the chamber transparent mylar window. A low counting rate ${ }^{241} \mathrm{Am}$ alpha source was placed in the chamber, just over the drift electrode, the $5.48 \mathrm{MeV}$ alpha particles of ${ }^{241} \mathrm{Am}$ have a range of $3.42 \mathrm{~cm}$ in Ar. The CCD images of the projections of the tracks alpha particles obtained with this chamber are shown in Fig. 10 and the corresponding Bragg curves in Fig. 11. We estimated that using a F1.8 $50 \mathrm{~mm}$ photographic lens placed $30 \mathrm{~cm}$ from the GEM plane we could collect around 180 photons per deposited $\mathrm{keV}$ in the gas [20].

Using a triple GEM setup in a detector chamber prepared for thermal neutron imaging filled with ${ }^{3} \mathrm{He}$ plus $\mathrm{CF}_{4}$ to control the range of the recoil of the nuclei, it is possible to image the recoil patterns of the proton and the triton, as has been suggested in [21]. The neutron capture in ${ }^{3} \mathrm{He}\left({ }^{3} \mathrm{He}+\mathrm{n} \Rightarrow \mathrm{p}+\right.$ $\left.{ }^{3} \mathrm{H}+770 \mathrm{keV}\right)$, releases a $579 \mathrm{keV}$ proton and a $193 \mathrm{keV}$ triton with ranges of $4.4 \mathrm{~mm}$ and $1.6 \mathrm{~mm}$ at 1 bar $\mathrm{CF}_{4}$, tracks using 400 mbar of $\mathrm{CF}_{4}$ should be about $15 \mathrm{~mm}$. An AmBe source with polyethylene shielding was used and the CCD images of the projections of the proton and triton tracks in ${ }^{3} \mathrm{He}-40 \% \quad \mathrm{CF}_{4}$ are shown in Fig. 12. The projection of the light intensity along the track as measured by the $\mathrm{CCD}$, highlighting the Bragg curves of the proton and triton [22], is shown in Fig. 13.

\section{Other applications}

Imaging chambers can be used for digital radiography using X-rays [23] and, taking advantage of the modern back illuminated CCDs that are sensitive down to $200 \mathrm{~nm},{ }^{1}$ mixtures scintillating in the upper UV with high penning effect [24] can be used to develop high-pressure Xe detectors using GEMs, overtaking the drawback of the low density of the gaseous media and low efficiency at energies higher than $10 \mathrm{keV}$. We built and tested such a detector using two GEMs in a pressurized chamber with a filling of 5 bar Xe with the addition of $2.5 \%$ TMA that was found to be the optimum quencher concentration for simultaneous maximum charge gain and luminosity. We could record CCD images of a radiography of a small snail $\sim 8 \mathrm{~mm}$, using a molybdenium X-ray tube at 30 and $40 \mathrm{kV}$ (Fig. 14). The position resolution of this system was checked with a collimated X-ray beam (anode voltage $30 \mathrm{kV}$ ) and was found to be approximately $50 \mu \mathrm{m}$ [25]. The Xe-TMA highpressure mixture was also studied for operation in pulsed mode using a photomultiplier and was found to be quite adequate for photon counting readouts, as light signals had a rise time of $60 \mathrm{~ns}$ and the energy resolution at $22.1 \mathrm{keV}\left({ }^{109} \mathrm{Cd}\right)$ was better than $20 \%$ at 3 bar.

GEMs readout in light mode can be used for dose imaging in proton radiotherapy [26]. Fig. 15 shows relative photon intensity of different scintillators at the Bragg peak using $138 \mathrm{MeV}$ protons,

\footnotetext{
${ }^{1}$ S7170 CCD package, Hamamatsu Photonics KK, Japan.
} 


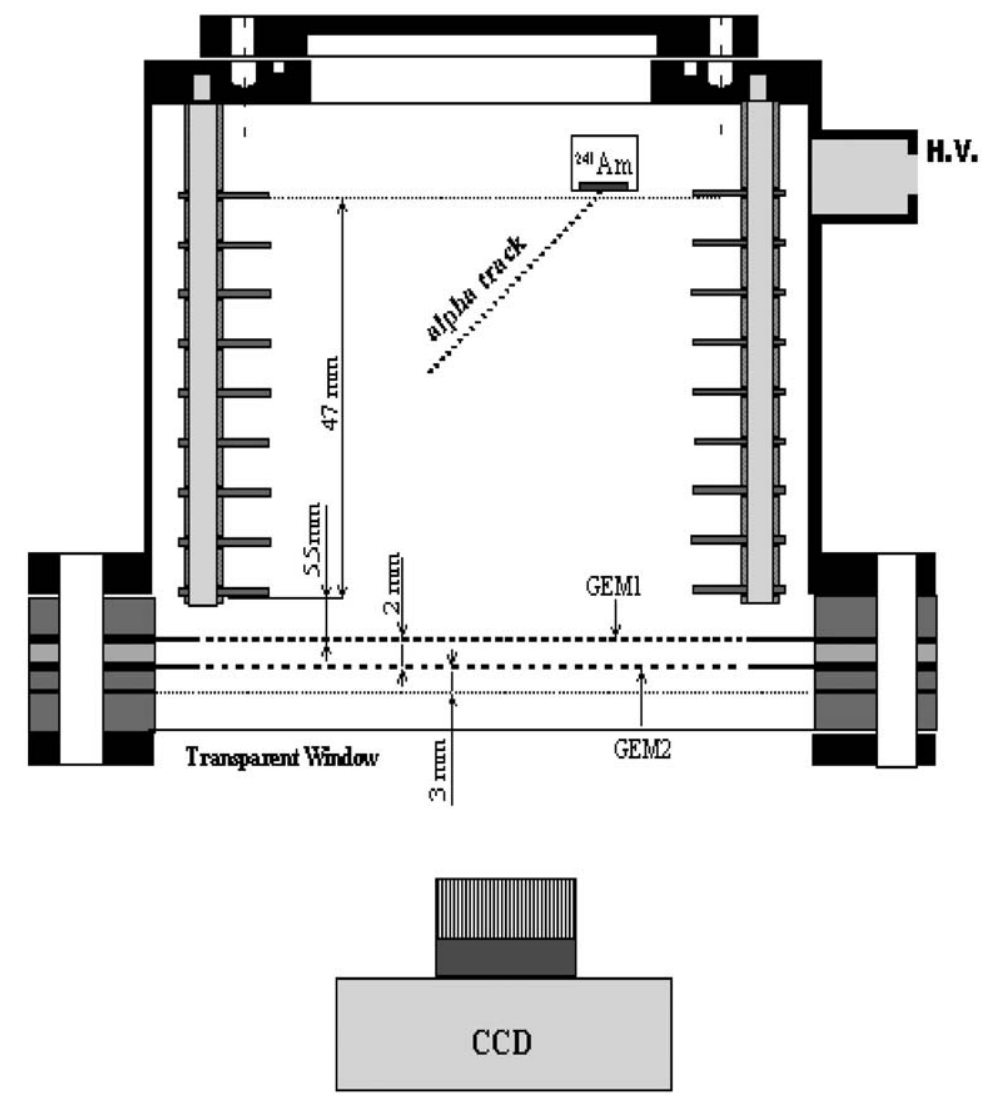

Fig. 9. Schematic cross-section of the tracking chamber used in this work. The CCD (not drawn to scale) was placed $30 \mathrm{~cm}$ away from the mylar window. The chamber has been operated with three cascaded GEMs, only two are shown in this picture.

where the GEM based device shows much better linearity than the currently used plastic scintillators that suffer from severe quenching at high energy density deposits [27].

\section{The future}

Extensive spectroscopic studies of the light emitted by the GEM avalanches have qualified us to have a better understanding of the fundamental aspects of scintillation in the UV, visible and NIR and to broaden the field of application of this technique. GEMs operated with an optical readout are currently being considered to several new applications. We would like to refer some of them.
Recoil neutron spectrometer for fast neutron spectroscopy (1-20 MeV) with single event energy resolution: The energy of the recoil target will be measured from the amount of scintillation and the direction of emission will be computed from the estimated real length of the track and the length of the projection. Efficiency is expected to be 10-100 better than current Li foil detectors.

Thermal neutron imaging: Solid converter detector with GEM active scintillator readout. This project is currently under appreciation by some groups integrating the TECHNI collaboration.

GEM polarimeter with optical readout: The information about the X-ray polarization can be extracted from the GEM images of the tracks of the primary electrons readout by CCDs or arrays of PMTs. 


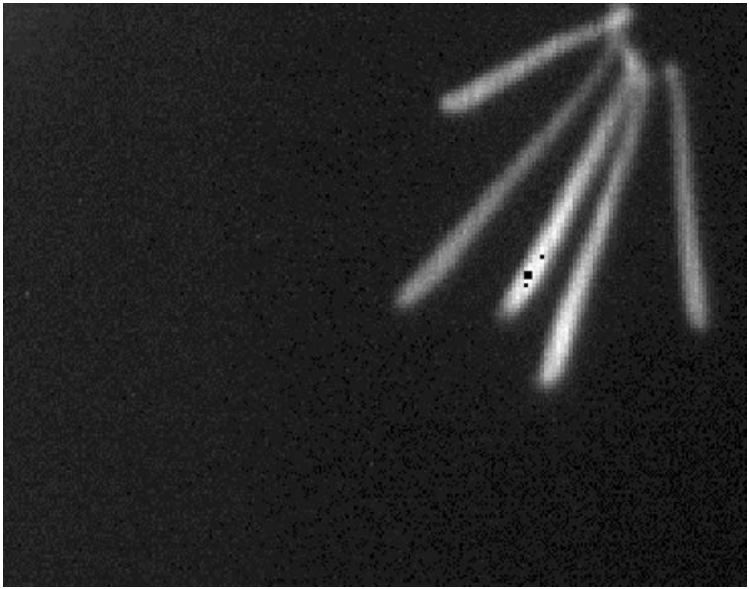

Fig. 10. CCD images of alpha tracks taken with a triple GEM tracking chamber with $\mathrm{Ar}-5 \% \mathrm{CF}_{4}$.
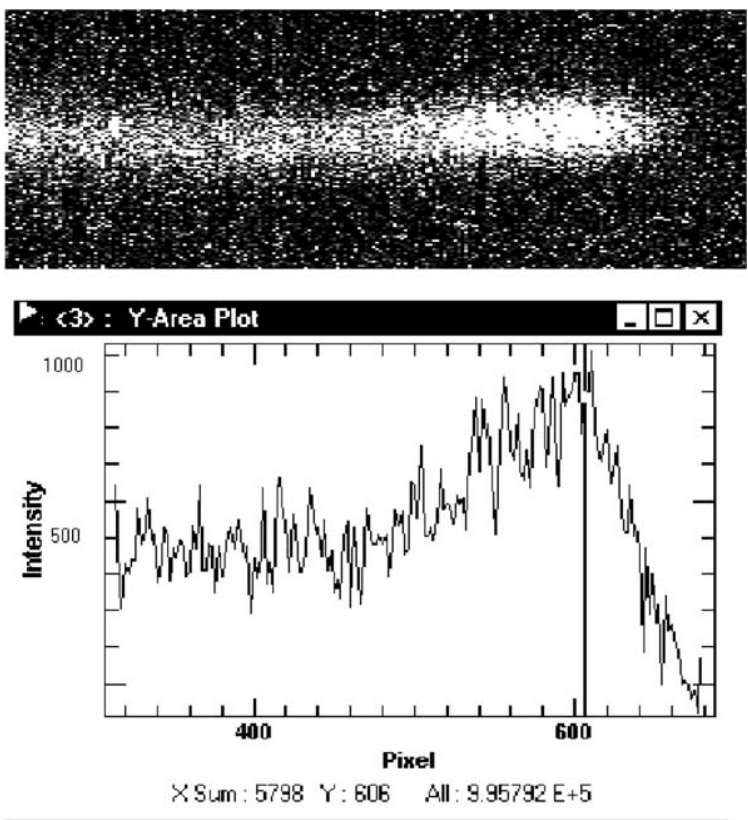

Fig. 11. Distribution of measured scintillation along an alpha track. The Bragg curve is revealed.

Optical readout of the DRIFTII TPC: This very low-diffusion TPC will be used to image nuclear recoils for dark matter search and GEMs operated in scintillation mode are being considered by the UK Dark Matter Collaboration for its readout.

GEMs coated with p-terphenyl: When coated with a suitable material, the GEM can work as an

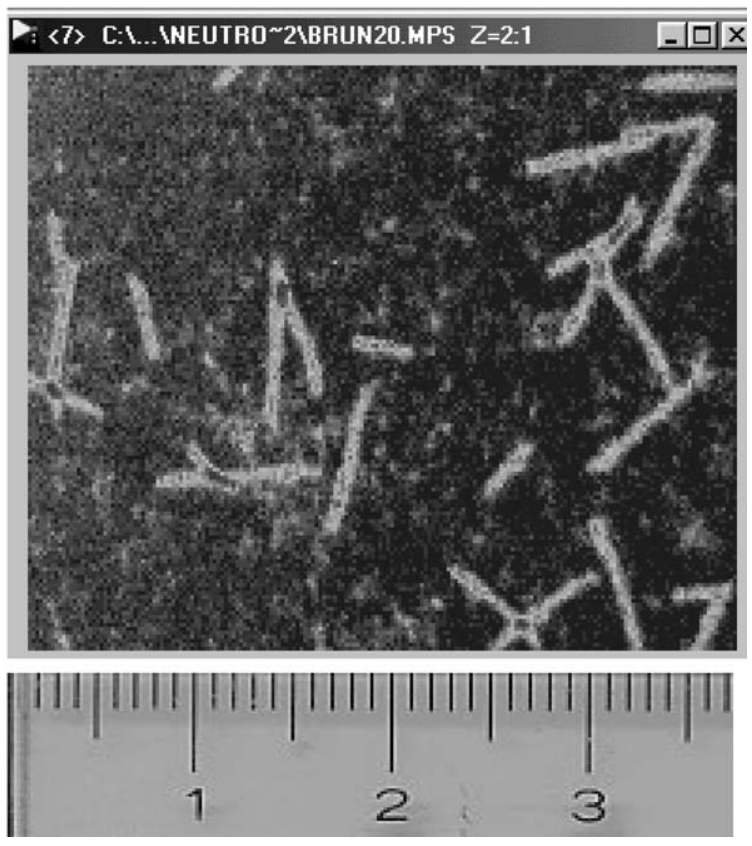

Fig. 12. Superimposed proton-triton linear tracks obtained with an exposition time of $1 \mathrm{~s}$. The gamma background (short marks) are also clearly seen.

active wavelength converter, without position resolution degradation due to diffusion of the UV light.

\section{Conclusion}

Optical readout of detectors will benefit from the huge improvements foreseen in the field of optical sensors in then next years. It is expected that the current limitation of CCDs, slow readout, will be improved by up to three orders of magnitude, due to faster techniques and improved architectures within a few years, and the first available avalanche photodiode arrays are now coming out, as well as CMOs imagers. Although these devices can present an alternative to PMTs, unfolding some very attractive characteristics, such as low volume and mass, and, once steady production is reached, low cost, they have a drawback when compared with PMTs - very low or no gain. We believe that gaseous active scintillators, with their high scintillation yields, 

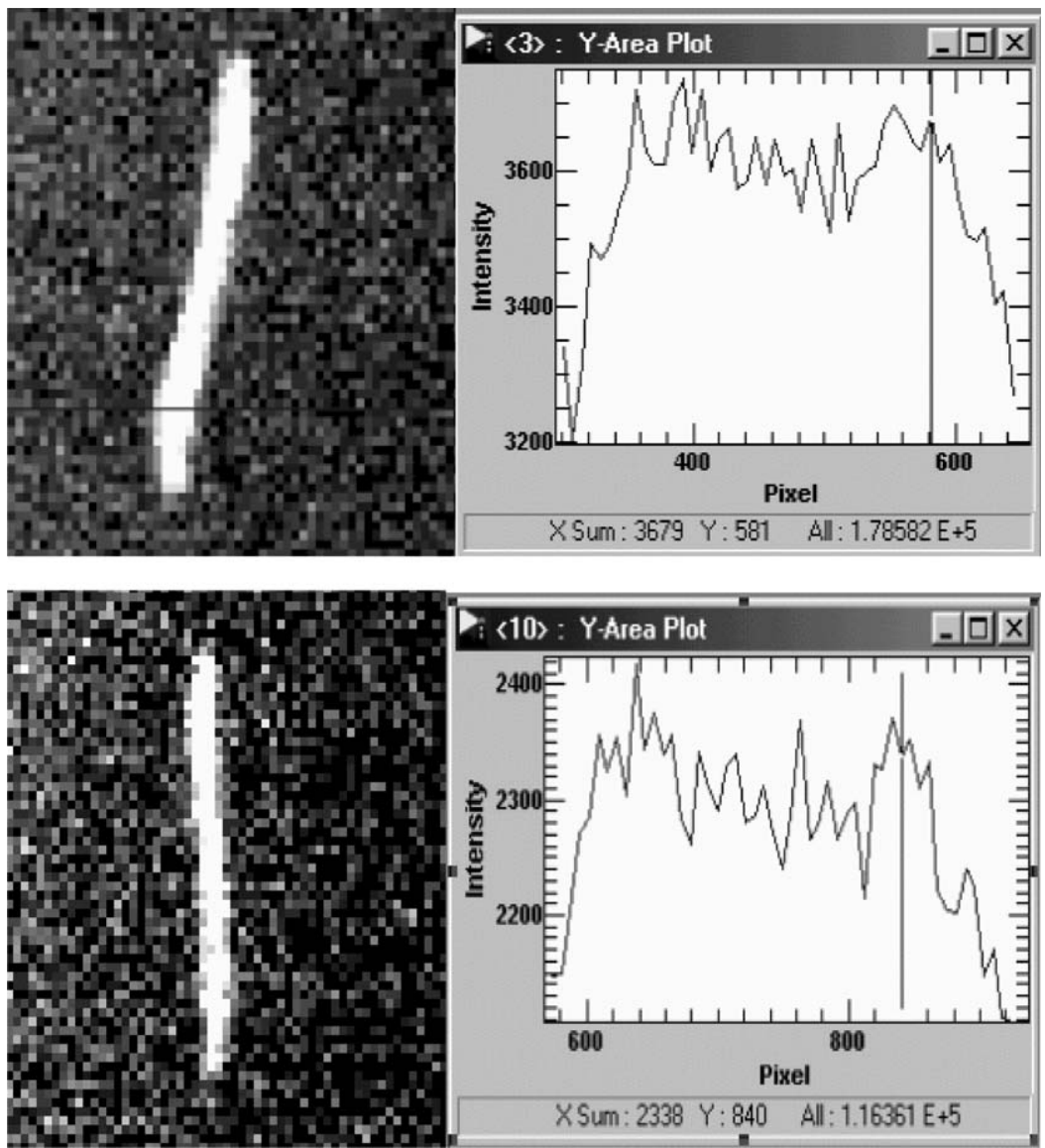

Fig. 13. Distribution of measured scintillation along proton-triton tracks. The Bragg curves of the proton (left) and triton (right) are revealed.
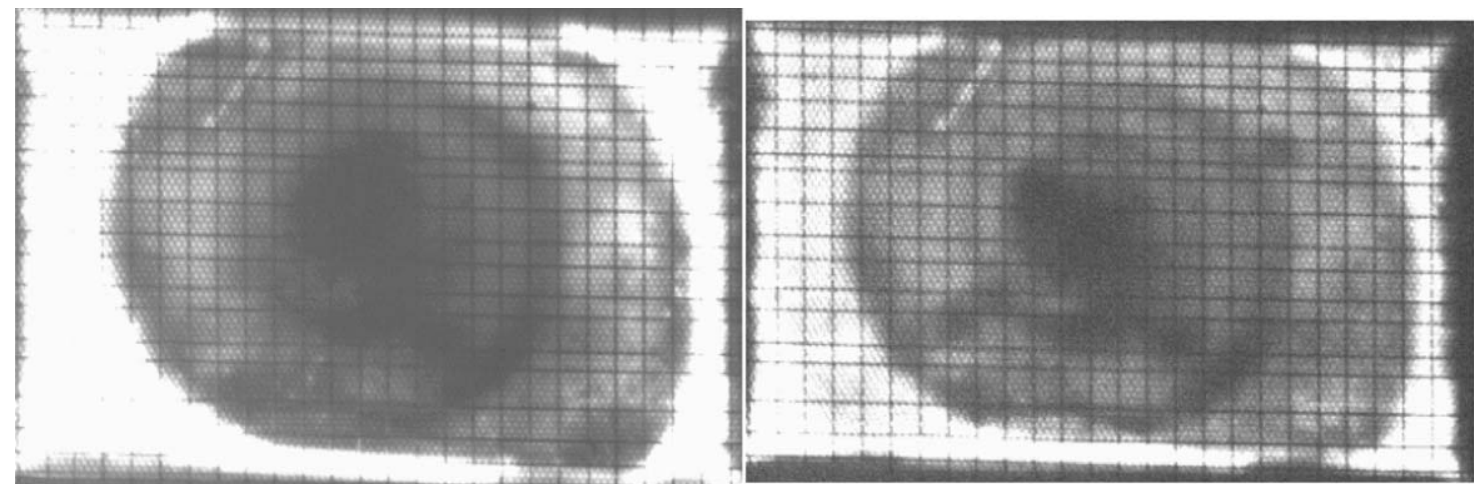

Fig. 14. Radiography of a small snail $(\sim 8 \mathrm{~mm})$ taken with a double GEM chamber filled with Xe-2.5\%TMA at 5 bar. A molybdenium X-ray tube was used at 30 and $40 \mathrm{kV}$ and the scintillation of the XeTMA was recorded by a backthinned UV CCD. 


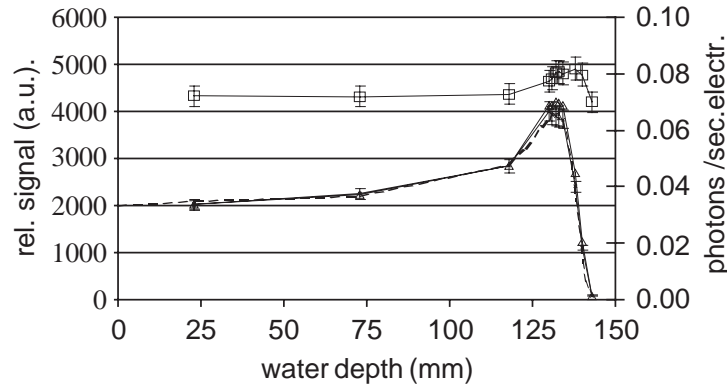

Fig. 15. Left scale: Measurement of the dose of $138 \mathrm{MeV}$ protons as a function of the water depth between source and detector with an ion chamber (- - ) and with the double GEM, operating at $V_{\mathrm{GEM} 1,2}=450 \mathrm{~V}$ and filled with $\mathrm{Ar}+10 \% \mathrm{CF}_{4} . I_{\text {out }}$ $(-)$ and the CCD signal $(-\Delta-)$ are normalized to the dose at $50 \mathrm{~cm}$ depth. On the right scale $\mathrm{L}(-\cdot-)$ is plotted as a function of depth.

typically two or three orders of magnitude better than their equivalent passive devices, will have an important part in optical detector development in the next years.

\section{Acknowledgements}

Current work on GEM luminescence is supported by the contract CERN/FNU/43735/2001 with the Portuguese FCT.

\section{References}

[1] A. Breskin, Nucl. Instr. and Meth. A 498 (1989) 457.

[2] G. Charpak, G.A. Renard, J. Phys. Radium 17 (1956) 585.

[3] A.J.P. Policarpo, Space Sci. Instrum. 3 (1977) 77.

[4] A. Breskin, R. Chechik, D. Sauvage, Nucl. Instr. and Meth. A 286 (1990) 251.

[5] G. Charpak, W. Dominik, J.P. Fabre, J. Gaudaen, F. Sauli, M. Suzuki, Nucl. Instr. and Meth. A 269 (1988) 142.

[6] V. Peskov, G. Charpak, W. Dominik, F. Sauli, Nucl. Instr. and Meth. A 227 (1989) 547.

[7] U. Titt, A. Breskin, R. Chechik, V. Dangendorf, H. Schmidt-Böcking, H. Schuhmacher, Nucl. Instr. and Meth. A 416 (1998) 85.

[8] T. Masuda, H. Sakurai, Y. Inoue, S. Gunji, K. Asamura, IEEE Trans. Nucl. Sci. NS-49 (2002) 553.
[9] P. Geltenbort, A. Oed, Proceedings of the Workshop on Progress in Gaseous Microstrip Proportional Chambers, Grenoble, 21-23 June 1993.

[10] F. Sauli, Nucl. Instr. and Meth. A 386 (1997) 531.

[11] F. Sauli, GEM: GAS ELECTRON MULTIPLIER, http:// gdd.web.cern.ch/GDD/

[12] L.M.S. Margato, F.A.F. Fraga, S.T.G. Fetal, M.M.F.R. Fraga, R. Ferreira Marques, A.J.P.L. Policarpo, B. Guerard, G. Manzin, Presented at the Beaune 2002 Conference on New Development in Photodetection, Nucl. Instr. and Meth. A 504 (2003) 374.

[13] F.A.F. Fraga, S.T.G. Fetal, R. Ferreira Marques, A.J.P.L. Policarpo, Nucl. Instr. And Meth. A 442 (2000) 417.

[14] A. Vàvra, A. Sharma, Nucl. Instr. and Meth. A 478 (2002) 235.

[15] D. Mörmann, A. Breskin, R. Chechik, P. Cwetanski, B.K. Singh, Nucl. Instr. and Meth. A 478 (2002) 230.

[16] A. Bondar, A. Buzulutskov, L. Shekhtman, A. Vasiljev, Nucl. Instr. and Meth. A (2003), these Proceedings.

[17] R. Chechik, et al., Nucl. Instr. and Meth. A 419 (1998) 423.

[18] B. Ketzer, S. Bachmann, M. Capeáns, M. Deutel, J. Friedrich, S. Kappler, I. Konorov, A. Placci, K. Reisinger, L. Ropelewski, L. Shekhtman, F. Sauli, CERN-OPEN2002-004.

[19] M.M. Fraga, F.A.F. Fraga, S.T.G. Fetal, L.M.S. Margato, R. Ferreira Marques, A.J.P.L. Policarpo, Presented at the Beaune 2002 Conference on New Development in Photodetection, Nucl. Instr. and Meth. A 504 (2003) 88.

[20] F.A.F. Fraga, L.M.S. Margato, S.T.G. Fetal, R. Ferreira Marques, A.J.P.L. Policarpo, IEEE Trans. Nucl. Sci. NS49-4 (2002) 281.

[21] G. Charpak, K.W. Dominik, J.P. Fabre, J. Gaudean, F. Sauli, M. Suzuki, Nucl. Instr. and Meth. A 269 (1988) 142.

[22] F.A.F. Fraga, L.M.S. Margato, S.T.G. Fetal, M.M.F.R. Fraga, R. Ferreira Marques, A.J.P.L. Policarpo, B. Guerard, A. Oed, G. Manzini, T. van Vuure, Nucl. Instr. and Meth. A 478 (2002) 357.

[23] F.A.F. Fraga, L.M.S. Margato, S.T.G. Fetal, R. Ferreira Marques, A.J.P.L. Policarpo, Nucl. Instr. and Meth. A 471 (2001) 125.

[24] T. van Vuure, C.W.E. van Eijk, F. Fraga, R.W. Hollander, IEEE Trans. Nucl. Sci. NS-48-4 (2001) 1092.

[25] L. M. S. Margato, F.A.F. Fraga, M.M.F.R. Fraga, S.T.G. Fetal, R. Ferreira Marques, A.J.P.L. Policarpo, T.L. van Vuure, R. Kreuger, C.W.E. van Eijk, R.W. Hollander, Presented at the Symposium on Applications of Particle Detectors in Medicine, Biology and Astrophysics II, Nucl. Inst. and Meth., submitted.

[26] J.H. Timmer, T.L. van Vuure, V. Bom, C.W.E. van Eijk, J. de Haas, J.M. Schipers, Nucl. Instr. Meth. A 478 (2002) 98.

[27] S.T.G. Fetal, C.W.E. van Eijk, F.A.F. Fraga, J. de Haas, R. Kreuger, T.L. van Vuure, J.M. Schippers, Nucl. Instr. and Meth. A, (2003) these Proceedings. 